\title{
Development of an Acquisition Instrumentation Program
}

\author{
Luisa A. Dempere ${ }^{1}$, Michele Manuel $^{2}$ \\ ${ }^{1 .}$ Research Service Centers, Herbert Wertheim College of Engineering, University of Florida, \\ Gainesville, USA. \\ 2. Department of Materials Science and Engineering, Herbert Wertheim College of Engineering, \\ University of Florida, Gainesville, USA.
}

Grant proposals for instrument acquisition are a heavily contended area of funding. Instruments with new capabilities come to the market regularly and those researchers that can use new technologies have the opportunity to collect and publish unique data. On the other hand, it is a daunting exercise to decide when existing technologies have advanced enough to justify instrument replacements or significant upgrades.

A clearly defined program around the acquisition of an instrument and its associated techniques is an approach to instrument procurement easier to embrace by proposal reviewers of funding agencies. A program implies a much larger vision and approach than moving existing capabilities to or closer to the state-of-the-art. It encompasses not only the need of the instrument for significant research purposes but it should also cover the education and training opportunities that brings to the students, the local and regional impact of access to the instrument, and the development of instrument enabled collaborations across disciplines and institutions (Figure 1).

The instrument program we proposed intended to bring together a unique set of 22 researchers from five state universities (Figure 2) and five science and engineering fields (Materials Science and Engineering, Mechanical and Aerospace Engineering, Geology, Chemistry, Physics) that had one commonality: the need to understand the connection between chemistry and structure at the sub-micron scale.

Research activities were described that addressed characterization and analysis of materials with an instruments that provided a unique integration of data difficult to correlate with existing instrumentation. The instrument was intended to combine wavelength dispersive spectroscopy (WDS) mapping with electron backscatter diffraction (EBSD) mapping in a single instrument. A prototype of the system existed and was ready to be available on a commercial basis.

The central theme of the instrumentation program concerning education and outreach was based on lowering institutional barriers. It was proposed to provide education through a remote access system that was expected to reach over 200 students every semester both on and off campus. The students expected to benefit the most from this effort included those taking it for continuing education and those in the military, as well as students registered at the university residing in other countries.

Remote access capabilities were also proposed to be used to collaborate with senior personnel from four different state universities. These activities were already taking place with out-of-state facilities taking away resources allocated to the state universities. Collaborators had also plans to use the online capabilities in their undergraduate courses. 


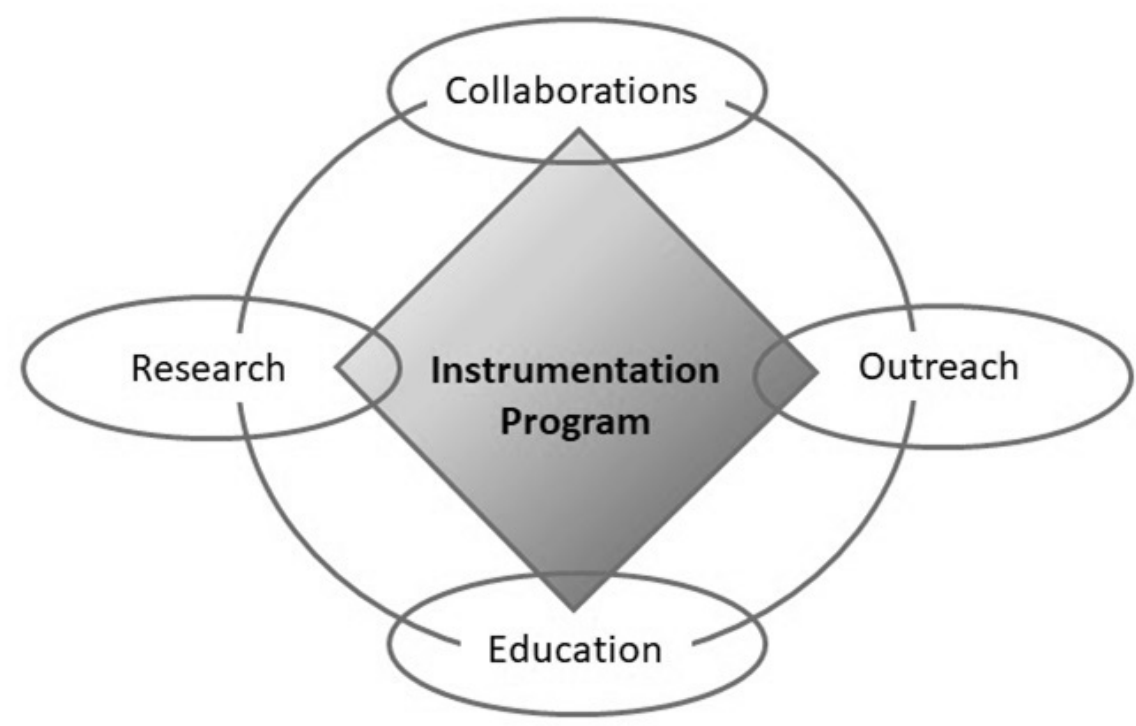

Figure 1. Diagram representing the interacting components of an instrument program proposal.

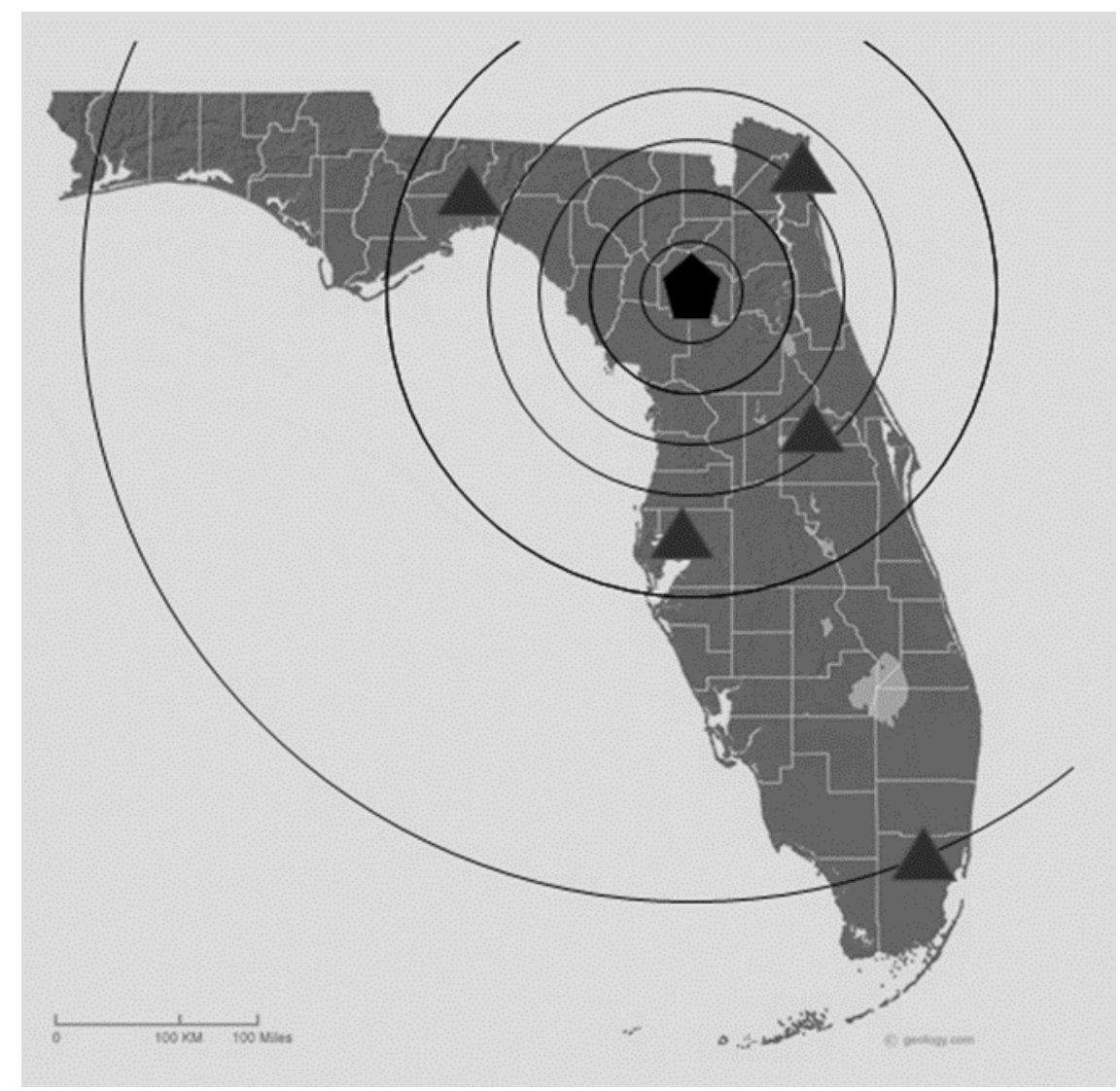

Figure 2. Map showing collaborating universities brought together under the instrument program and the remote access capabilities that enable distance interactions. 